\title{
The Double Embeddedness of Bitcoin: Insights from Old and New Economic Sociology
}

\author{
Fiammetta Corradi $^{1}$ \\ ${ }^{1}$ University of Pavia, Italy \\ Correspondence: Fiammetta Corradi, University of Pavia, Italy \\ Received: April 25, 2018 \\ Accepted: May 17, 2018 \\ Available online: May 18, 2018 \\ doi:10.11114/ijsss.v6i6.3289 \\ URL: https://doi.org/10.11114/ijsss.v6i6.3289
}

\begin{abstract}
Revisiting analytically the notion of embeddedness and its connections with the concept of trust, this paper shows that contrary to Bitcoin's premises and promises to be a trust-low or even trust-less currency, trust enters the system at many various levels and with different nuances. Applying a conceptual framework that conceives embeddedness as both the possible source and outcome of trust, it is pointed out that Bitcoin should better be regarded as doubly embedded: in technology and in its peculiar social structure. Due to the existence of computational and cognitive asymmetries within the system, in fact, trust is necessary for the very functioning of this new form of money, as well as for its future prospects.
\end{abstract}

Keywords: embeddedness, bitcoin, blockchain, mining pools, trust, rationality, economic sociology

\section{Introduction}

In a "White paper" appeared on the internet in 2008, the mysterious founding father of Bitcoin - known under the pseudonym of Satoshi Nakamoto - presented it as "an electronic payment system based on cryptographic proof instead of trust, allowing any two willing parties to transact directly with each other without the need for a trusted third party" (p. 1$)^{\mathrm{i}}$.

Since the appearance of that revolutionary manifesto, Bitcoin has widely been considered as a trust-low or even as a trust-less currency, a form of money designed and supposed to function without any kind of trust. This assumption has been often uncritically embraced and emphasised by Bitcoin's advocates, as one of the main pillars of the libertarian ideology supporting this new form of money.

In the present work I challenge the soundness of this view and I develop a conceptual framework that should help seeing how and where in the Bitcoin system trust comes in. I strongly believe, in fact, that the acknowledgement of the various roles that trust plays in the system will not only provide a better understanding of Bitcoin's functioning (and current success), but also to recognize some of the intrinsic, potential threats to the system itself.

To argue that the consideration of Bitcoin as a trust-less currency is deeply unsound, I first revisit the notion of "embeddedness", one of the key notions in both classical and contemporary economic sociology. After having shown that the conceptual link between "embeddedness" and trust has been underestimated in literature at risk of loosing some of its heuristic value ( $§ 2)$, I discuss analytically the notion of trust through the pioneering insights contained in the Philosophy of Money by George Simmel (1900), to re-think embeddedness as both possible source and outcome of trust (§ 3). I then apply this conceptual framework to the Bitcoin system, coming to the conclusion that Bitcoin should better be conceived as doubly embedded, both in technology (§ 4) and in social structure (§ 5), with trust having multiple roles and "nuances" in backing and supporting this so far successful, but certainly not trust-free, cryptocurrency.

\section{On "Embeddedness"}

Few concepts have become so distinctive of a discipline as the one of "embeddedness" in the field of economic sociology, bridging the classical tradition to the so-called "new economic sociology" (from the 1980s onward). This holds true notwithstanding its alleged polysemous meaning, varying on the author and sometimes even on different contexts of usage by the same author.

In the history of economic sociology, "embeddednmess" has been a key theoretical tool to discuss and assess the degree, the extent and the ways in which economic action is entangled, embodied, incorporated or rooted into social structures, 
and eventually to re-settle the disciplinary boundaries between economics and sociology. A brief account of its usages would probably help the reader to contextualize my own theoretical approach to the problem of embeddedness and its connections to the notion of trust.

Notoriously, the Hungarian scholar Karl Polanyi was the one who made popular the concept of embeddedness to signal the strong and reciprocal intertwinement between economic actions and the social system. In his masterpiece - The Great Transformation, 1944 - summing up the main problem with liberal capitalism, Polanyi wrote: "Instead of the economic system being embedded in social relationships, these relationships were now embedded in the economic system". In Polanyi's view, such unleashed self-regulating market represented the latest stage of development in the historic evolution of the relation between economy and society. In fact, in another work (Primitive, Archaic and Modern Economies, 1968), he identified the so-called three forms of integration/organization of the economy, using the degree of "incorporation" of economic exchanges into social or political norms as the crucial criterium divisionis. Accordingly, he described the historical process of the progressive up-rooting of economic action in three distinct stages: from pervasive and shared moral and social norms (in the form named "reciprocity"), from the political normative system (in the form named "redistribution"), and finally from any kind of social structure (in the form of the "self-regulating market"). Only in this latter stage the economic system (and its laws) became "visible" for social scientists as an independent object of study (in this way, Polanyi also explained why economics was born so late compared to other sciences).

According to attentive observers in the field of economic sociology (Swedberg, 2003), Polanyi's legacy remained essentially forgotten or latent for some decades, until Mark Granovetter published his much quoted work Economic Action and Social Structure: The Problem of Embeddedness (1985). That article placed embeddedness at the centre of a new theoretical approach, becoming the milestone for a new research programme.

I personally believe that the fascination of the idea of embeddedness after Mark Granovetter's contribution mainly ${ }^{\text {ii }}$ stemmed from its deep connection with the ancient and debated problem of social order (already portrayed by Hobbes in The Leviathan). As Granovetter brilliantly argues, the two main solutions to such riddle - how can it be that self-interested, opportunistic individuals behave most of the times correctly from an economic viewpoint, without systematically recurring to force and fraud - have been radically alternative. On the one hand, exponents of utilitarianism and of neoclassical economics have embraced an under-socialized view of man and economic action, based on an atomized conception of decision-making, claiming that only the existence (or the creation) of a super-individual authority (or of an authoritative institutional arrangement) can tame the innate opportunism of economic agents, leading them out of a "state of nature", where homo homini lupus. On the other hand, many sociologists, but also eclectic economists like Polanyi, have favoured an over-socialized view of man and economic action, where people, due to socialization processes, are "overwhelmingly sensible to the opinion of others", have largely internalized social norms and where the problem of order is supposed to be solved trough a "generalized morality". Both solutions, according to Granovetter, are inadequate because they wrongly neglect the crucial role of social relations and of networks of social relations in building reciprocal trust: a non economic condition of possibility for any economic action, and more generally for social life.

One may agree with B. Uzzi in thinking that this notion of embeddedness is very abstract, as far as it "lacks its own concrete account of how social relations affect economic exchange" (Uzzi, 1997. p. 35) ${ }^{\text {iii }}$. In any case it is certainly among Granovetter's greatest merits to have first hinted at some conceptual connection between the concept of embeddedness and the one of trust: a link that has mostly been neglected and not further specified in the dedicated literature, where the efforts have focused instead on the quest for more precise definitions of embeddedness ${ }^{\text {iv }}$.

Consequently, I propose to maintain a very general notion of embeddedness (the abstraction level could be the one proposed by Polanyi and implicitly shared by Granovetter) and at the same time to explore analytically the concept of trust, in order to identify their conceptual ties. The pioneering insights about confidence provided by George Simmel in the Philosophy of Money, 1900, will help me to show that embeddedness could be considered both as source and outcome of trust.

\section{An Analytical Inquiry in the Concept of Trust}

According to its libertarian background (for extensive details on Bitcoin's ideology and Hayek's legacy see Ametrano, 2016), Bitcoin has the declared ambition to solve the so-called double spending problem, the challenge of warranting that the same unit of a currency cannot be spent twice (or even more times), without the intermediation of any third party: being it a political institution (for instance, the National State) and/or a central bank (such as the FED or the EBC). This twofold "de-intermediation" ambition is probably at the basis of the claim that, consequently, Bitcoin is the first currency finally free from any form of trust. To start showing to what extent and why this claim is false, I first report Simmel's too often forgotten words about trust in money. 
"[...] money transactions would collapse without trust. There are nuances of this trust. The assertion that money is always credit because it rests upon the recipient's confidence that he will be able to acquire a certain quantity of goods in exchange for it is not entirely adequate. For it is not only a money economy, but any economy, that depends upon such trust. If the farmer did not have confidence that his field would bear grain this year as in former years, he would not sow; if the trader did not believe that the public would desire his goods, he would not provide them etc. This kind of trust is only a weak form of inductive knowledge. But in the case of credit, of trust in someone, there is an additional element which is hard to describe: it is mostly clearly embodied in religious faith. When someone says that he believes in God, this does not merely express an imperfect stage of knowledge about God, but a state of mind which has nothing to do with knowledge, which is both less and more than knowledge. To 'believe in someone', without adding or even conceiving what it is that one believes about him, is to employ a very subtle and profound idiom. It expresses the feeling that there exists between our idea of a being and the being itself a definite connection and unity, a certain consistency in our conception of it, an assurance and lack of resistance in the surrender of the Ego to this conception, which may rest upon particular reasons, but is not explained by them. Economic credit does contain an element of this supra-theoretical belief, and so does the confidence that the community will assure the validity of the tokens for which we have exchanged the products of our labour in an exchange against material goods. This is largely, as I have said, a simple induction, but it contains a further element of socio-psychological quasi-religious faith" (Simmel, 1990; 1978. p. 179).

Of course I'm not quoting this passage to belabour the obvious, namely, that since Bitcoin is considered as a form of money (for specific objections to this point see Wenzel, 2013 and Yermack, 2013), and Simmel is claiming that there cannot exist any money without trust, hence, Bitcoin cannot be considered as a trustless currency. This would be a trivial (but nonetheless problematic ${ }^{v}$ ) syllogistic reasoning.

Instead, I believe that three distinct theoretical insights should be retained from this long extract: once analytically split and commented upon they will result heuristic in understanding Bitcoin more deeply.

The first tenet highlights the key role of trust, as a condicio sine qua non - of a non-economic nature - in any economic action. Already at this level of analysis, considering money as a medium of exchange, trust is presented by Simmel as twofold. On the one hand, one must be confident that a certain coin (or token) has indeed the value it claims to have. On this regard Simmel further writes: "cash transactions cannot develop without public confidence in the issuing government or perhaps in those persons who are able to determine the real value of the coin in relation to its nominal value" (p. 178). Hence, proposing a somewhat forgotten possible alternative to a centralized, political warranting instance, Simmel hints at the collective or social acknowledgment of value to money. Analogously, social acknowledgement is necessary to confer some value to Bitcoin and to assess its value as a medium of exchange. On the other hand, Simmel underscores how important it is that there be confidence in the stability of such value over time. He restates this point, recalling the inscription on the coins of Malta, in these terms: "what is indispensible and conclusive is non aes sed fides - the confidence in the ability of an economic community to ensure that the value given in exchange for an interim value, a coin, will be replaced without loss" (ivi).

If the reader looks at Figure 1, showing Bitcoin's price volatility since its market appearance in 2009, more than one doubt could rise about the stability of Bitcoin value as a medium of exchange over time (the price soared from less than $\$ 30$ to more than $\$ 19,200$ in less than 10 years, with a surprising acceleration since 2017!)

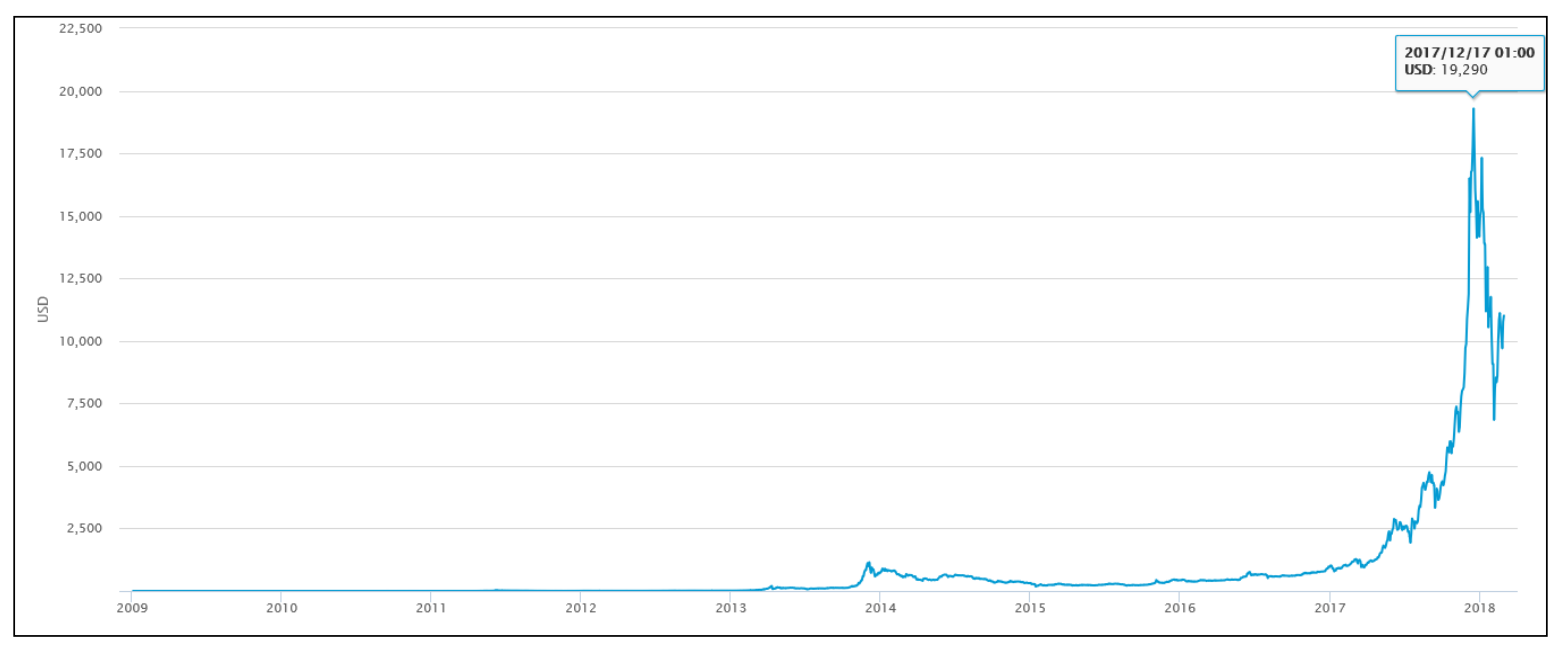

Figure 1. Average USD market price across major bitcoin exchanges, since 2009 
Inizio modulo

Source: https://blockchain.info/de/charts/market-price?timespan=all

A second theoretical contribution to the understanding of the essence and role of trust regards its double nature, rational and non-rational (although Simmel does not use these categories). The rational dimension entails "a weak form of inductive knowledge", a knowledge based on idiosyncratic inferences from past experiences (like the one backing the farmer's expectation that his crop will provide a return as it did in former years) ${ }^{\mathrm{vi}}$. The non-rational dimension of trust is instead identified with a "quasi-religious faith", and it is presented as the "feeling that there exists between our idea of a being and the being itself a definite connection and unity". Here is where the notion of coherence comes in, fostering the leap of faith that trusting someone or somewhat entails, with its emotionally reassuring side-benefits ${ }^{\mathrm{vii}}$, but also with all the alleged risks.

This second analytical distinction somehow reflects the more recent elaborations of trust in rational choice theory terms (or as such I propose to read it). James Coleman, for instance (1990), reframes the idea of trust as "a rational bet"viii , where actors would be theoretically able to foretell the expected payoffs of their trust-choices on the basis of past experiences (in the case of iterated games). In this way, I suggest, Coleman works on what Simmel christens a "weak form of induction", leaving aside or behind the "quasi-religious faith". More or less along the same line, Bacharach and Gambetta (2001) add valuable insights to the signalling theory splitting two different levels in which (rational) confidence comes in: in trusting the person of $\mathrm{X}$ to be able to accomplish $\mathrm{Y}$ and in trusting eventual signs that support such "weak form" of induction. In this way, they propose a further divide, namely between personal/interpersonal trust and its cognitive grounds, placing emphasis on the rational dimension of trust. More generally, rational choice theorists have preferred to focus on the cognitive determinants of trust, leaving aside the "leap of faith" dimension, while other sociologists have maintained the original Simmellian ambivalence (see for instance Möllering, 2001, who also provides a detailed discussion of Simmel's legacy in Luhmann and Giddens),

The third and last (but not least) tenet derivable from the extract is Simmel's conceptual positioning of trust in an intermediate place between knowledge and ignorance. If one had complete and certain knowledge, he would not need trust; if one had no knowledge at all, trust would turn into blind faith, loosing its rational dimension (the weak form of induction). Moreover, this location of trust in a middle position between certainty and ignorance helps explaining why personal as well as institutional trust ${ }^{\mathrm{ix}}$ is always risky, and as such it is reasonable to expect it to be warranted.

Through this articulated conceptualization of the notion of trust it is now possible to see the "double bind" connecting trust and embeddedness. If we agree, following Polanyi and Granovetter's legacy, that economic action is to various degrees and in various ways embedded into social structures (which could only be assessed empirically), then such embeddedness is conceptually upstream and downstream of economic action. In other words, embeddedness can be seen as a source of trust for economic action; on the other hand it can also be the outcome of economic actions based on trust.

Equipped with this conceptual framework I can now proceed to show that Bitcoin displays a double form of embeddedness and - consequently - depends on trust for its existence and functioning much more that it is generally believed.

\section{The Technological Embeddedness of Bitcoin}

As I suppose known, Bitcoin's overall system is a digital permission-less distributed network relying, for the solution of the already mentioned double-spending problem, on the technology named "blockchain" (whose practical applications are now spanning well beyond the realm of cryptocurrencies ${ }^{\mathrm{x}}$ ).

The blockchain is generally described as the public ledger that allows the validation of each transaction and the record of formal agreements between parties without the intermediation of any external third party ${ }^{\mathrm{xi}}$. Within such supposedly horizontal structure, where there is no centralized authority, and the nodes are not hierarchically disposed as client and server, the log is effectively public for everybody, but it is not constructed collectively by every Bitcoin buyer (seller). Instead, the blockchain is built by some of the nodes/users, named "miners", who provide the computational power of their pc to the network and assemble the transactions occurred in the last 10 minutes in a file termed "block". In this way, miners (or rather, mining pools, as I will explain below) check the validity and uniqueness of transactions in bitcoins and make them public (receiving in exchange a payoff).

More specifically, to warrant the validity of each block, the system uses a cryptographic protocol based on the algorithm SHA-256, that makes the validation of a block a mathematical problem whose solution implies increasing levels of difficulty. The difficulty changes automatically every 2016 blocks and it has reached skyscraper levels, such that already in 2013 only enormous pc, as huge as wardrobes, were able to solve the mathematical problem. Since computational and energetic powers are costly, mining activity is designed to reward the fastest, effectively mining performers with a certain number of bitcoins as payback, currently 12.5 BTC (which conveys some intrinsic value to 
bitcoins, contrary to those who claim it has no intrinsic value at all).

Already from this synthetic and oversimplified description of the system, one should note that 1) Bitcoin is embedded, for its functioning, in mathematical and computational devices; 2) there are computational - and cognitive asymmetries among the nodes and more generally among the Bitcoin' share and stakeholders.

The first consideration recalls some kind of institutional/systemic trust, in the sense that, to take part in the system, one has to have confidence in the overall set of technical and automatic rules designed to solve the double-spending problem. The technological embeddedness of Bitcoin, in other words, mainly functions as a source of trust. The second consideration, instead, suggests that the Bitcoin system is embedded in a very peculiar social structure where trust is qualitatively different, this time pertaining to (and emerging from) social interactions. To this second form of embeddedness is now time to turn our attention.

\section{The Social Embeddedness of Bitcoin}

I'm not certainly the first sociologist to highlight the social dimension of the Bitcoin system. Niel Dodd (2017), for instance, has proposed a very brilliant analysis of the social practices of Bitcoin, singling out one of the most interesting paradoxes underpinning the whole system ${ }^{\text {xii }}$. Differently from Dodd, however, I would distinguish between two different meanings in which Bitcoin is socially embedded. The first concerns the uses of Bitcoin as a speculative asset. The second pertains to the system social structure and to the existence of cognitive and computational asymmetries within the system. Since this second aspect has been comparatively less investigated than the first, I will explore the second in deeper details.

Data and statistics prove beyond doubt that bitcoins have been and constantly are treated (and traded) as speculative assets in intraday transactions. This speculative appeal is probably fostered by the astonishing Bitcoin's price volatility (already displayed in fig. 1) ${ }^{\mathrm{xiii}}$. At the same time, Bitcoin's volatility has ignited sharp critiques by economists, multiplied the warnings about potentially disruptive "speculative bubbles" and refreshed the notion of "irrational exuberance" (echoing the title of a popular book by Shiller, 2000).

It certainly goes beyond this paper purposes to assess whether or not such euphoria for Bitcoin since 2017 is (or will prove to be) "irrational". To my argumentation line it will suffice to notice that phenomena of financial euphoria (and panics), as well as other kinds of financial contagion, could be considered as examples of sudden, collective excesses (or defects) of confidence in a given asset. What is relevant here is to highlight that such contagious behaviors pushing people to buy (or sell) assets when the prices are not justified by fundamental values - are typically social in their origin. As I have extensively explained in a dedicated book (Corradi, 2016), building on John Maynard Keynes' seminal insights about the role of social expectations in the stock markets, many of the "irrationalities" that financial markets cyclically witness are undesired and "perverse" effects of "words of mouth", imitation strategies, reactions to winning advertisement strategies (like advertising bitcoin on platforms for hazard games), rather than of the (often dramatic) outcome of sophisticated hoaxes such as Ponzi schemes.

The second meaning in which Bitcoin is socially embedded refers to the social structure of its network, which is inherently competitive, with mining pools contending on computational power to dig new bitcoins. The computational and cognitive asymmetries among nodes within the system derive straight from Bitcoin's thick embeddedness in technology and from the evolution of technological devices over time.

At the beginning of Bitcoin's life (2009), the difficulty in solving the Bitcoin algorithm (SHA-256) was in fact very low, requiring limited computational and energy power: "solo-miners", at that time, were the majority. As the computational power needed to solve the algorithm and to add validated blocks to the blockchain grew exponentially, "solo-miners" progressively left the floor to groups (pools) of miners with variable computational poweriv (they can also be conceived as extraction companies). This process has been catalyzed by the introduction of new technical devices, more and more advanced and fast, but at the same time increasingly expensive. The CPU (Central Processing Units, the main chip in a pc to perform all tasks) to which Nakamoto makes reference in his manifesto are now considered completely out of date in the field of cryptocurrencies mining and they have been substituted first by GPUs and then by the so called ASIC based processors (Application Specific Integrated Circuit). So, basically, mining pools are groups of miners that unite their computational power - measured in Hashrate ${ }^{\mathrm{xv}}$ - to increase the overall one of the pool and to split the costs for the purchase and running of cutting edge technological devices (as well as the payoffs) ${ }^{\mathrm{xvi}}$.

As Beikverdi and Song have already underscored, this kind of cooperation requires some degree of interpersonal trust:

"joining a mining pool requires some levels of trust for each individuals, in the network that is claimed to be purely trust less. Additionally some of these mining pools offer services as cloud mining. Cloud mining refers to services in which anyone can buy or rent a mining hardware from their server facility and these cloud pools pay them accordingly to their contracts. Hence in this case individual miners who rent or buy these hardware are not aware of the hashing 
processes and it is all under pools control" (Beikverdi \& Song, 2015)

Within the mining activities, however, trust plays a key role also at the more socially aggregated level of competing mining pools. Actually, individuals active in cloud mining would be better off if they had any idea of the overall hashrate distribution in the system. This kind of information is daily provided in the form of estimations by a dedicated website (https://blockchain.info/pools) that also displays valuable quantitative data about the overall system. So, for instance, a 4 days average distribution retrieved on March 2018 (Fig. 2), shows that there is one major mining pool (BTC.com), which alone owns more than $25 \%$ of the overall computational effectiveness, followed by four major competitors (AntPool, ViaBTC, SlushPool and BTC.TOP), with shares ranging from 16,6\% to 9,4\%. 13 other mining pools share the remaining hashrate.

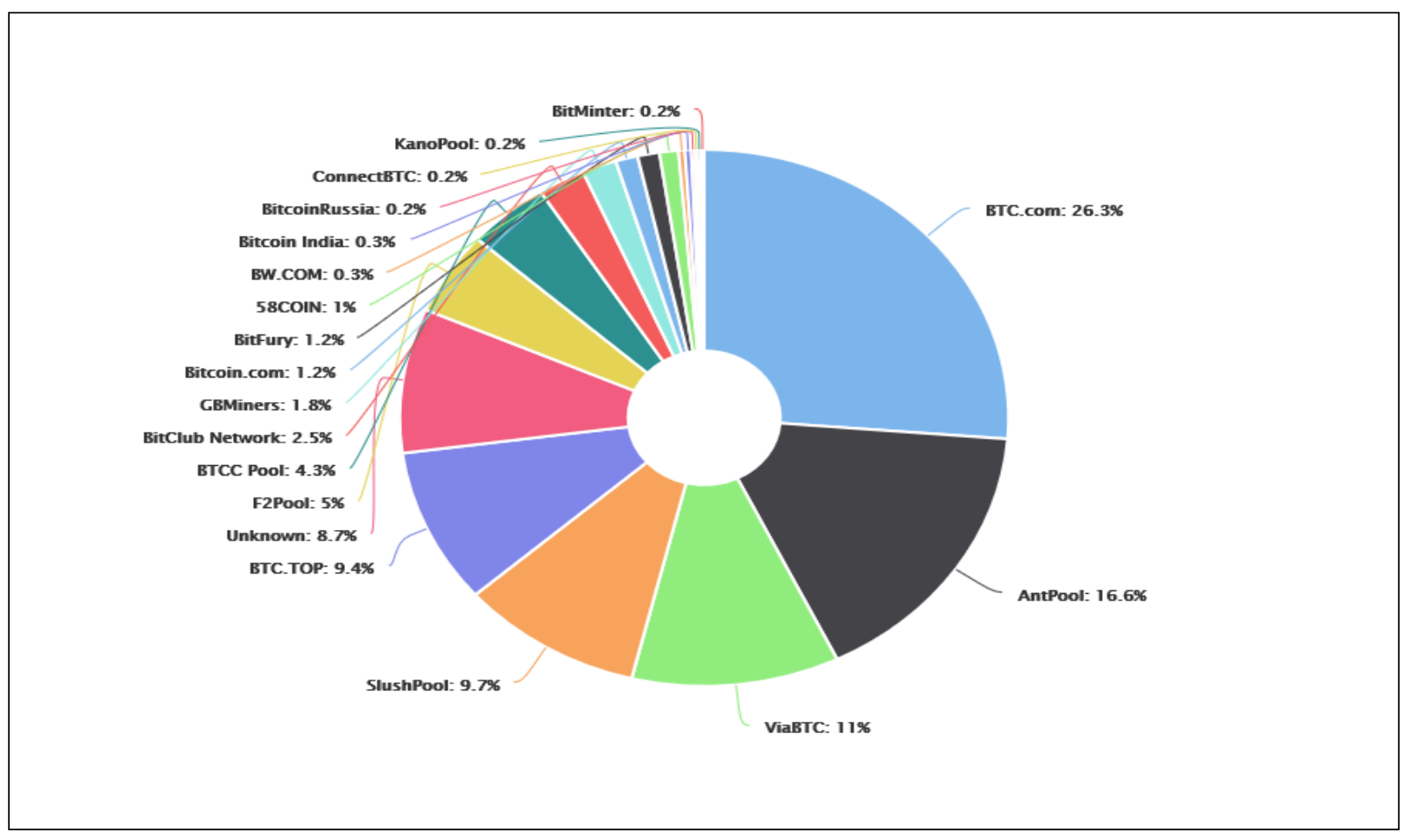

Figure 2. An estimation of Hashrate distribution (4 days av., 8-12/03/2018)

\section{Source: https://blockchain.info/pools}

Comparing these estimated distributions with the ones retrieved in 2016 by N. Shi (2016. p. 2), it is possible to note that in a relatively short time span (about 2 years) the computational power of some mining pools has grown at a marveling rate, while the one of others has restrained significantly (see for instance F2Pool, from 13\% to 5\%). At the same time, while the rate un "unknown" hashrate was much smaller in 2016, the number of small competing pools was notably greater than today. These comparative data, although certainly insufficient and in need of future completion, seem to suggest that, due to the increase in computational difficulty (and alleged costs), the current competitive pluralism could leave the floor to M\&A between stronger mining pools, leading towards higher degrees of centralization (eventually until a monopolistic assessment). In any case, these structural transformations do not change the underlying embeddedness of Bitcoin in social structure, neither devalue the key role of trust at this aggregation level (although I would probably rephrase it in terms of reputation rather than in terms of trust).

Future, additional empirical research along this path - which is so far only tentative and exploratory - would probably show that mining pools' reputation (trust) matters in changing the hashrate distribution within the bitcoin system, making some mining pools stronger and pushing others out of the competition. If this conjecture would turn out to be verified, we would dispose of a very interesting example of how embeddedness could as well be the outcome of trust, not only its source.

\section{Conclusive Remarks}

In this work I have argued that Bitcoin's ambition to be a totally dis-embedded and trust-less currency is unsound. Revisiting analytically the concepts of embeddedness and trust, I have proposed to view embeddedness as both possible source and outcome of trust. Moreover, trough an analytical approach to the notion of trust, I have singled out different 
dimensions and types of confidence. Such articulated conceptual framework has then been applied to the Bitcoin'system to show that it is deeply embedded: in technology and in a competitive social structure.

Hence, contrary to Bitcoin's premises (and promises), trust enters the system at many various levels and with different nuances (for the existence of computational and cognitive asymmetries), contributing to its functioning and - so far - to its appalling success. At the same time, the acknowledgement of Bitcoin's double embeddedness highlights potential threats to the system and warns about some possible endogenous changes.

This paper finally sketches a future research path in the competitive social structure of Bitcoin, that promises to provide an interesting empirical example of how embeddedness could also be the outcome of trust (or reputational) relations, not only its source.

\section{References}

Ametrano, F. A. (2016). Hayek Money: the Cryptocurrency Price Stability Solution. Retrieved from https://doi.org/10.2139/ssrn.2425270

Bacharach, M., \& Gambetta, D. (2001). Trust in Signs. In K. S. Cook (ed.), Trust in Society (pp. 148-184). New York: Russell Sage Foundation series on Trust, Volume II.

Baker, W. E. (1984). The social structure of a national securities market. American Journal of Sociology, 89(4), 775-811. https://doi.org/10.1086/227944

Barber, S., Boyen, X., Shi, E., \& Uzun, E. (2012). Bitter to better. How to make bitcoin a better currency. In A. D. Keromytis (Ed.) Financial Cryptography and Data Security, 16th international conference (pp. 399-414). Berlin: Springer.

Beikverdi, A., \& Song, J. S. (2015). Trend of Centralization in Bitcoin's distributed network. 16the IEEE/ACIS International Conference on Software Engineering, Artificial Intelligence, Networking and Parallel/Distributed Computing (SNPD), 00, 1-6. https://doi.org/10.1109/SNPD.2015.7176229

Böhme, R., Christin, N., Edelman, B., \& Moore, T. (2015). Bitcoin: Economics, technology, and governance. Journal of Economic Perspectives, 29(2), 213-238. https://doi.org/10.1257/jep.29.2.213

Capoti, D., Colacchi, E., \& Maggioni, M. (2015). Bitcoin revolution. La moneta digitale alla conquista del mondo. Milano: Hoepli.

Coleman, J. (1900). Foundations of Social Theory. Cambridge, Mass. London: The Belknap press of Harvard University press.

Cook, K. S. (Ed.) (2001). Trust in Society, New York: Russell Sage Foundation, Russell Sage Foundation Series on Trust, Volume II.

Corradi, F. (2016). Social Spirits, A new research agenda for the Sociology of the Stock Exchange. Saarbrücken, Germany: LAP LAMBERT Academic Publishing.

Corradi, F., \& Höfner, P. (2018). The disenchantment of Bitcoin: Unveiling the Myth of a Digital Currency. International Review of Sociology, 28(1), 193-207. https://doi.org/10.1080/03906701.2018.1430067

Davis, G. F., \& Greve, H. R. (1997). Corporate elite networks and governance changes in the 1980s. American Journal of Sociology, 103(1), 1-37. https://doi.org/10.1086/231170

Dodd, N. (2017). The Social Life of Bitcoin. Theory, Culture and Society, First published online December 17, 2017; 0(0), 1-22; Issue published: May 1, 2018, 35(3), 35-56. https://doi.org/10.1177/0263276417746464

Granovetter, M. (1985). Economic action and social structure: The problem of embeddedness. American Journal of Sociology, 91(3), 481-510. https://doi.org/10.1086/228311

LaMarsh, I. (2017). Bitcoin: What it is, How it works, and why it is giving the world a run for its money. Oxford Journal: An International Journal of Business \& Economics, 12(2), 1-10. http://www.ojbe.org/oj/index.php/journals/article/view/133

Mauri, L., Cimato, S., \& Damiani, E. (2018). A comparative analysis of Current Cryptocurrencies. Proceedings of the 4th International Conference of Information Systems Security and Privacy (ICISSP 2018), pp. 127-138. https://doi.org/10.5220/0006648801270138

Möllering, G. (2001). The Nature of Trust: From Georg Simmel to a Theory of Expectation, Interpretation and Suspension. Sociology, 35(2), 403-420. https://doi.org/10.1177/S0038038501000190

Nakamoto, S. (2008). Bitcoin: A Peer-to-Peer Electronic Cash System. Retrieved from https://bitcoin.org/bitcoin.pdf 
Polanyi, K. (1944). The great transformation: The political and economic origins of our time. Boston: Beacon Press.

Polanyi, K. (1968). Primitive, Archaic and Modern economies, Essays of Karl Polanyi, Edited by George Dalton, Garden City, New York:Anchor Books.

Romano, D., \& Schmid, G. (2017). Beyond Bitcoin: A Critical Look at Blockchain-Based Systems. Cryptography, 1, 15, 1-31. https://doi.org/10.3390/cryptography 1020015

Shi, N. (2016). A new proof-of-work mechanism for bitcoin. Financial Innovation, 2(31). https://doi.org/10.1186/s40854-016-0045-6

Simmel, G. (1900). Die Philosophie des Geldes. Berlin: Duncker \& Humbolt Verlag; The Philosophy of Money, ed. 1978, London: Routledge \& Kegan Paul.

Swedberg, R. (2003). Principles of Economic Sociology, Princeton: Princeton University Press.

Tasca, P. (2015, September 7). Digital Currencies: Principles, Trends, Opportunities, and Risks, UCL, Centre for Blockchain Technology. https://doi.org/10.2139/ssrn.2657598

Uzzi, B. (1997). Social structure and competition in interfirm networks: The paradox of embeddedness. Administrative Science Quarterly, 42, 35-67. https://doi.org/10.2307/2393808

Uzzi, B. (1999). Embeddedness in the making of financial capital: How social relations and networks benefit firms seeking financing. American Sociological Review, 64, 481-505. https://doi.org/10.2307/2657252

Wenzel, R. (2013). Is Bitcoin Money? What Economists Have To Say. Economic Policy Journal. Retrievable from http://www.economicpolicyjournal.com/2013/10/is-bitcoin-money-what-economists-have.html

Yermack, D. (2013). Is Bitcoin a real currency? An economic appraisal. Technical report 19747, retrievable from National Bureau of Economic Research, http://www.nber.org/papers/w19747.pdf; then published in David K.C. Lee (ed.), The Handbook of Digital Currency, pp. 31-44, Elsevier, 2015. https://doi.org/10.3386/w19747

${ }^{\mathrm{i}}$ More details on the mysterious character of Satoshi Nakamoto and on the history of Bitcoin in Corradi and Höfner (2018).

ii Of course part of its attractiveness also derives from its fitness to exploration through the methodological approach and instruments of network analysis.

iii I also believe that this limit has only been partly overcome by Granovetter's own empirical works using network analysis, as well as some by Uzzi, $(1997 ; 1999)$ despite his interesting effort to differentiate components of embedded ties.

iv Many conceptual efforts have actually been devoted to operationalize the concept of embeddedness in order to explain various economic phenomena, from the relations between price volatility and socio-cultural patterns (Baker, 1984), to governance and partners choices (Davis \& Greve, 1997).

v I call it problematic because Bitcoin's features as a digital currency to some extent fail to fulfil Simmel's own definition of money. For more details on this point see Tasca (2015).

${ }^{v i}$ Slightly different interpretations of the supposed "weakness" of this inference can be imagined. This induction can be dubbed as "weak" because it is empirically rooted into the likelihood (instead of into the certainty) of repetition over time of similar events; alternatively, because it is based on the somehow unchecked assumption that what held in the past will hold in the future as well (a variant of the fallacy of generalization).

${ }^{\text {vii }}$ From a psychological viewpoint, this second insight suggests that a variety of cognitive mistakes (like self-deception, wishful thinking and others) could be by-products of the human tendency to avoid or correct instances of cognitive dissonance, so re-establishing the reassuring feeling of coherence.

viii Coleman writes: “( $(.$.$) the elements confronting the potential trustor are nothing more or less than the considerations a$ rational actor applies in deciding whether to place a bet" (1990. p. 99).

${ }^{\text {ix }}$ Here I refer to the classical distinction in the sociological literature between institutional/systemic trust (or confidence) and interpersonal trust, which draws a useful divide between trust in systems of rules and trust in the ability of $\mathrm{X}$ to do $\mathrm{Y}$, or in his/her honesty (see Cook, 2001).

${ }^{x}$ For an interesting overview see Romano and Schmid (2017).

${ }^{x i}$ For more details: Barber et al. (2012); Böhme et al. (2015); Capoti et al. (2015); LaMarsh (2017); Mauri et al. (2018).

xii Dodd phrases such paradox in the following terms: "Bitcoin will succeed as money to the extent that it fails as an ideology. The currency relies on that which it seeks to deny, namely, the dependence of money upon social relations, and 
upon trust" (Dodd, 2017, p. 3).

xiii In passing, intraday strategies are not necessarily alternative to keeping strategies that would be encouraged instead by the pre-fixed top-ceiling in bitcoin's overall supply (21 million units, expected to be reached by 2040).

${ }^{\text {xiv }}$ Investing in cloud-mining, one benefits from a remote hardware and from the experience of other miners and shares the profits on a contractual basis. For more details on the pros and cons and for a synthetic overview of the three main types of the cloud mining, see http://easymining.altervista.org/index.html.

${ }^{\mathrm{xv}}$ Mining power is calculated through a unit of measure termed "hashing". 1KH/s is 1,000 hashes per second, and it equals $1 \mathrm{MH} / \mathrm{s}$.

xvi Once a block has been successfully added by a mining pool, the joined "workers" share the rewards proportionally to their own computational power or through other possible methods (like the PPS, pay per share, or the POT, pay on target).

\section{Copyrights}

Copyright for this article is retained by the author(s), with first publication rights granted to the journal.

This is an open-access article distributed under the terms and conditions of the Creative Commons Attribution license which permits unrestricted use, distribution, and reproduction in any medium, provided the original work is properly cited. 